\title{
Conhecimento Organizacional: uma revisão conceitual de modelos e quadros de referência
}

\author{
Davi Noboru Nakano \\ Professor Doutor \\ Escola Politécnica da Universidade de São Paulo \\ Departamento de Engenharia de Produção \\ dnnakano@usp.br \\ Afonso C. C. Fleury \\ Professor Titular - Chefe de Departamento \\ Escola Politécnica da Universidade de São Paulo \\ Departamento de Engenharia de Produção
}

Com a crescente atenção que o estudo do conhecimento organizacional e de sua gestão tem recebido, existem diversas proposições, análises, modelos e quadros de referência disponíveis na literatura. Este artigo faz uma análise detalhada de alguns dos principais artigos da área, especialmente aqueles sintetizando as classificações desenvolvidas nesses trabalhos, bem como as dimensões e níveis de análise utilizados por eles. Ele tem como um de seus objetivos suprir a carência de revisões conceituais mais detalhadas em língua portuguesa. De fato, embora o assunto desperte grande interesse, são ainda poucos os trabalhos (em nossa língua) que recuperam e analisam em mais detalhe os textos mais significativos na área. Este artigo seleciona 12 proposições, oriundas de três correntes de pesquisa: Aprendizagem Organizacional, Visão da Empresa baseada no Conhecimento e Gestão do Conhecimento. Conclui-se que os estudos podem ser diferenciados de acordo com sua perspectiva, se cognitiva ou construtivista, e pelo seu caráter, se prescritivo ou analítico. A distinção permite organizar e entender com mais clareza a literatura da área, que embora farta, soa, em certos momentos, dissonante, tornando o seu estudo mais complexo.

Palavras-chave: conhecimento organizacional; aprendizagem organizacional; gestão do conhecimento.

Organizational knowledge and its management have received increased attention in the last years. As a result, there are a number of literature reviews, models and frameworks in the literature. This article analyses some of the most influential texts published to date, and synthesizes the classifications, dimensions and levels of analysis used by those pieces. It aims to fill a void in the Brazilian literature. Despite the interest on knowledge, there are currently very few com-prehensive studies. This papers takes twelve propositions, from three different research streams: Organizational Learning, Knowledge-based View of the Firm and Knowledge Management. One can find that they can be sorted according to their perspective, whether cognitive or constructive, and by their approach, whether prescriptive or analytical. The distinction allows a better understanding of the literature on knowledge management.

Keywords: organizational knowledge; organizational learning; knowledge management.

\section{Introdução}

A literatura sobre conhecimento organizacional é composta por diversas correntes, e por esse motivo é farta, tanto em número quanto em perspectivas e pontos de vista, o que acaba, em muitos casos, por se tornar problema, pois seu mapeamento e compreensão tornam-se complexos. Este artigo tem por objetivo contribuir para uma melhor compreensão conceitual da área, reunindo e comparando alguns dos principais modelos e quadros de referência publicados na literatura até o início desta década. Procurou-se colocar lado a lado os estudos clássicos na área com outros mais contemporâneos, e oferecer uma visão comparativa de suas propostas, sintetizando as diversas classificações para conhecimento, bem como as dimensões e níveis de análise utilizados. Focalizou-se em particular três correntes de pesquisa: uma mais tradicional, a Aprendizagem Organizacional, e duas mais contemporâneas, Visão da Empresa Baseada no Conhecimento (Knowledge-based view of the firm) e Gestão do Conhecimento. 
A Aprendizagem Organizacional figura entre as correntes de pesquisa mais antigas a estudar o conhecimento nas organizações, ao lado da Gestão da Tecnologia e da Cognição Gerencial (GRANT, 1996), e dentre as três, é a mais abrangente. A Gestão da Tecnologia, corrente de pesquisa que conta com cerca de 40 anos, estuda a administração da tecnologia e inovação dentro das organizações, e desenvolveu-se acompanhando a crescente mudança tecnológica que caracterizou os últimos anos (CYERT e KUMAR, 1994). É porém considerada uma corrente de foco restrito para estudos mais abrangentes sobre o conhecimento organizacional (HEDLUND, 1994), embora trabalhos recentes procurem estabelecer uma ponte entre conhecimento e assuntos já tradicionalmente estudados em gestão de tecnologia, como: localização e organização de laboratórios de P\&D e aprendizagem organizacional (LAM, 2003) e inovação de produtos (RONCHI et al., 2003), e indo ainda mais adiante, sistemas de gestão do conhecimento para P\&D (PARK e KIM, 2005). Da mesma forma que a corrente anterior, a Cognição Gerencial possui um foco bastante específico. Ela assume que os administradores das organizações são trabalhadores da informação que empregam seu tempo absorvendo, processando e disseminando informações (WALSH, 1995). A literatura sobre cognição gerencial possui uma linguagem própria (para uma extensa revisão bibliográfica, ver Walsh (1995)), e pode ser organizada em torno de três temas básicos: processos cognitivos, estilos cognitivos e estruturas de conhecimento (SCHNEIDER e ANGELMAR, 1993).

Já a Aprendizagem Organizacional é considerada, dentre as três, a mais abrangente e multidisciplinar, possuindo hoje uma vasta coleção de trabalhos (EASTERBY-SMITH et al., 1998). Entre as disciplinas que contribuem na sua formação estão: Psicologia e Desenvolvimento Organizacional, Gestão da Informação, Sociologia e Teoria das Organizações, Cultura Organizacional e Gestão de Operações (EASTERBYSMITH, 1997). Uma interessante revisão acerca de trabalhos sobre Aprendizagem Organizacional pode ser encontrada em Bontis et al. (2002). A Aprendizagem Organizacional é considerada uma corrente com fundamentos sólidos (HIRSCH e LEVIN, 1999) que ganhou espaço próprio e importância dentro da teoria organizacional (MINER e MEZIAS, 1996).

Seus conceitos têm se mostrado eficazes no estudo de diversos fenômenos relacionados às organizações. Por exemplo, foram usados por Fleury e Fleury (1995) para comparar as trajetórias de industrialização entre países como Japão, Coréia e Brasil, assim como as diferenças entre estratégias em alguns de seus setores industriais. Os mesmos autores (em FLEURY e FLEURY, 2000) voltaram a usar conceitos de Aprendizagem Organiza- cional e formação de competências para o estudo das novas configurações e estratégias adotadas pelas empresas transnacionais, o papel de suas subsidiárias no Brasil, e os posicionamentos estratégicos das empresas brasileiras face aos desafios da globalização. Outra aplicação do conceito é a controvérsia sobre Aprendizagem Organizacional dentro da Gestão de Operações entre Adler e Cole (1993) e Berggren (1993), que discordam sobre a superioridade do modelo japonês (defendida pelos primeiros) sobre o modelo sóciotécnico (defendido pelo último), como promotor de aprendizagem individual ou organizacional.

Algumas das raízes da Aprendizagem Organizacional podem ser encontradas nos trabalhos sobre teoria organizacional de March e Simon (1958). Esses autores salientaram a importância do conhecimento armazenado nos procedimentos internos de uma organização (SOP Standard Operational Procedures), estabelecendo assim um dos pontos de partida para a discussão sobre aprendizagem. Outro ponto de origem é o trabalho de psicólogos industriais como Argyris e Schön, cujo livro sobre Aprendizagem Organizacional (ARGYRIS e SCHÖN, 1978) foi um dos pioneiros na distinção entre cognição e comportamento no aprendizado.

A partir da última década do século passado, o interesse no conhecimento organizacional levou ao surgimento de outras correntes de pesquisa, entre elas a Visão da Empresa baseada no Conhecimento (Knowledge-based View of the Firm) (OLIVEIRA Jr., 2001) e a Gestão do Conhecimento (HEDLUND, 1994; NONAKA, 1994). Além dessas, outras correntes contemporâneas são a Inteligência Competitiva (KAHANER, 1996) e o Capital Intelectual (STEWART, 1997). A primeira é um caso particular da Visão da Empresa baseada em Recursos (RBV-Resource-based View of the Firm), corrente que se desenvolveu primariamente dentro da pesquisa em estratégia nas organizações. Seu conceito central é o de que organizações são conjuntos organizados de recursos, e que a posse de recursos raros e de difícil imitação confere, à organização que os possui, vantagem competitiva a longo prazo (OLIVEIRA Jr., 2001). Quando o recurso fundamental é o conhecimento, tem-se a Visão da Empresa baseada no Conhecimento (CONNER e PRAHALAD, 1996; GRANT, 1996).

Gestão do Conhecimento é uma corrente de pesquisa ainda em formação, que compartilha conceitos e apresenta convergência com a Aprendizagem Organizacional (EASTERBY-SMITH et al., 2000). De uma forma ampla, é o estudo de todos os processos relacionados à transferência e transformação do conhecimento dentro da organização (HEDLUND, 1994; TEECE, 1998). Sob uma ótica mais restrita, é definida como uma iniciativa que emprega tecnologia da informação para a gestão de informações dentro da organização, 
com ênfase no armazenamento e distribuição eletrônica de dados (EASTERBY-SMITH et al., 2000). Também é muitas vezes desagregada entre gestão do conhecimento operacional e estratégico (PABLOS, 2002).

É a partir da literatura das três correntes: Aprendizagem Organizacional, Visão da Empresa baseada no Conhecimento e Gestão do Conhecimento que se desenvolve este artigo. São analisadas as perspectivas, classificações e dimensões de análise para conhecimento organizacional utilizadas na literatura, que permitem uma visão dos processos relativos à aprendizagem e sua gestão, o que permite a síntese de um quadro com os principais processos de gestão do conhecimento explícito.

\section{Conceito e classificações do Conhecimento Organizacional}

É interessante notar que muitos trabalhos sobre Aprendizagem Organizacional nem mesmo mencionam explicitamente o conhecimento. Ele é assumido implicitamente como o resultado do processo de aprendizagem, sua decorrência natural. É por exemplo o caso de March e Olsen (1979), um dos mais influentes e citados artigos na área (CROSSAN et al., 1995). Seu foco é sobre as diversas possibilidades que podem levar uma organização a não realizar um ciclo completo de aprendizagem. $\mathrm{O}$ ciclo começa com os modelos mentais dos indivíduos que compõem a organização, usados na interpretação da realidade e tomada de ações. Como resultado das ações individuais, a organização, como a coletividade dos indivíduos, age, "escolhe" ou "decide". Da ação organizacional (coletiva) resulta uma resposta do ambiente, que é observada e interpretada pelos indivíduos, podendo levá-los a mudar seus modelos mentais, encerrando o ciclo. Quando esse ciclo é rompido em algum ponto, a organização deixa de apresentar um comportamento de adaptação racional. Diversas causas podem romper o ciclo, sendo uma das principais a ambigüidade das informações provenientes do ambiente, e a conseqüente dificuldade em interpretá-las.

Outro trabalho influente em Aprendizagem organizacional, o de Daft e Weick (1984), compartilha em certa medida o mesmo ponto de vista de March e Olsen (1979). Para esses autores o ambiente é extremamente complexo, gerando um volume tal de informações que seus dirigentes "literalmente devem vagar em um oceano de eventos" (p. 286, traduzido pelo autor). Organizações devem interpretar esse oceano de eventos para tomar decisões e articular planos de ação, apresentando diferentes comportamentos nesse processo. Novamente o conhecimento é um estado ou propriedade da organização em um dado instante, e a aprendizagem modifica esse estado ou propriedade ao longo do tempo.
Ainda entre os trabalhos mais antigos e influentes, Hedberg (1981) esposa o mesmo ponto de vista: conhecimento é ao mesmo tempo o estado ou propriedade de um sistema de aprendizagem e o seu próprio resultado. A noção de conhecimento como resultado da aprendizagem e estado/propriedade de uma organização está explícita ou implicitamente colocado em diversos trabalhos: nas revisões bibliográficas de Fiol e Lyles (1985) e Levitt e March (1988), nos modelos de sistemas de aprendizagem de Shrivastava (1983) e nas relações entre cultura organizacional e organização do trabalho com aprendizagem de Fleury e Fleury (1995).

Tomando uma linha ligeiramente diferente dos trabalhos anteriores, Huber (1991) identifica conhecimento com informação. Isso é evidente na maneira como Huber utiliza indistintamente informação e conhecimento como sinônimos, por exemplo ao afirmar: "muitas atividades organizacionais têm por finalidade adquirir informação ou conhecimento" (HUBER, 1991, p.91, traduzido pelo autor). A identificação de conhecimento com informação será extensamente explorada em trabalhos posteriores sobre Gestão do Conhecimento.

Boa parte da literatura sobre Aprendizagem Organizacional portanto identifica conhecimento como: (a) um estado ou propriedade de uma organização, e (b) o resultado de um processo de aprendizagem. Porém maior ênfase é dedicada ao processo de aprendizagem, e não ao seu resultado, o conhecimento. Essa posição começou a merecer algumas críticas a partir da década de 90, com a emergência da Visão da Empresa baseada no Conhecimento (OLIVEIRA Jr., 2001) e trabalhos explicitamente voltados para o estudo da Gestão do Conhecimento (e.g. HEDLUND, 1994; KOGUT e ZANDER, 1992; NONAKA, 1994). Kogut e Zander (1992) por exemplo argumentam "é curioso que a atenção considerável que foi dedicada a como as organizações aprendem tenham obscurecido o fato que as organizações ‘sabem' alguma coisa” (traduzido pelo autor). É principalmente a partir desses trabalhos que mais atenção é devotada para o conhecimento organizacional, através da discussão da sua natureza, e de suas classificações e tipologias.

Definir conhecimento não é tarefa fácil. Grant (1996) explica sua posição: "A questão tem intrigado alguns dos maiores pensadores do mundo, de Platão a Popper, sem o surgimento de um consenso claro, essa é uma arena que escolho não adentrar" (p.110, traduzido pelo autor). Dessa forma, muitos autores não definem conhecimento explicitamente, mas propõem classificações que revelam aspectos de sua natureza. Kogut e Zander (1992), por exemplo, dividem-no em informação e knowhow, Grant (1996), em knowing how e knowing about e Moorman e Miner (1997), em conhecimento procedural 
e declarativo, mesma clasificação seguida por Kyriakopoulos e de Ruyter (2004).

Uma classificação do conhecimento das mais populares é a baseada no trabalho de Polanyi sobre o conhecimento tácito (e.g. POLANYI, 1997). Hedlund (1994) e Nonaka (1994), por exemplo, fazem uso dessa classificação, dividindo o conhecimento em duas categorias: tácito e explícito (ou articulado). Hedlund (1994) subdivide cada uma das duas categorias de conhecimento (tácito e explícito) em três formas: conhecimento cognitivo (modelos e preceitos mentais), habilidades e o conhecimento incorporado em produtos e serviços. Porém, não se atém à análise dessas formas de conhecimento, pois seu foco está menos nas definições e mais sobre a dinâmica de transformação entre uma e outra forma de conhecimento. Por esse mesmo motivo, Hedlund (1994) utiliza informação e conhecimento como conceitos intercambiáveis, embora reconheça que análises mais detalhadas necessitem fazer distinção entre os dois conceitos.

Oliveira Jr. (2001), em sua revisão de literatura, resgata a classificação de conhecimento tácito de Spender, que o distingue em três componentes: o consciente, o automático e o coletivo. Oliveira Jr. (2001) também oferece uma relação entre a classificação de Kogut e Zander (1992) e a de Hedlund (1994) e Nonaka (1994), afirmando: "parece clara a relação que pode ser estabelecida entre informação e conhecimento explícito, por um lado, e know-how e conhecimento tácito, por outro lado" (p. 134). Recupera ainda a relação estabelecida por Grant (1996), entre o "saber como" (knowing how) e o conhecimento tácito, e o "saber sobre" (knowing about) e o conhecimento explícito. Castillo (2002) elabora sobre quatro tipos de conhecimento tácito: o emotivo, que não pode de forma nenhuma ser expresso em palavras; o sócio-cultural, ou seja, aquele que é parte do contexto social e cultural do indivíduo; o semântico, que é verbalizado através de palavras com alto grau de simbolismo; e o sagaz, que reflete a habilidade de apreender rapidamente a forma e caráter de um evento pela sua simples observação. O quadro da Figura 1 resume as diferentes classificações apresentadas e suas relações.

Embora o quadro organize algumas das principais classificações utilizadas para o conhecimento organizacional, e sugira que existe convergência entre elas, é importante ressaltar que existem visões bastante diferentes, que partem da premissa que o conhecimento tem "natureza dual e contraditória" (SCHULTZE e STABELL, 2004, p. 550, tradução do autor). Por exemplo, Schultze e Stabell (2004) utilizam os conhecidos paradigmas de Burrell e Morgan, originariamente desenvolvidos para classificar as vertentes em Teoria das Organizações, para produzir uma classificação para o conhecimento. Para evitar uma possível dissonância, pois esses trabalhos representam uma linha bastante diversa da adotada aqui, que inclusive critica e discorda de alguns dos trabalhos analisados neste texto, essas discussões não serão incluídas, o que não significa que elas não sejam consideradas importantes.

\section{Dimensões e níveis de análise do Conhecimento Organizacional}

Kogut e Zander (1992) definem duas dimensões de análise do conhecimento organizacional: codificabilidade e complexidade. A primeira dimensão mede o grau com que ele pode ser transformado em um conjunto de regras e relações, i.e., codificado, que possam ser facilmente comunicadas. A segunda, a complexidade, sugere o grau de esforço necessário para efetuar essa transfor-

\begin{tabular}{|c|c|c|}
\hline & Conhecimento Explícito & Conhecimento Tácito \\
\hline Sinônimos & $\begin{array}{l}\text { - Informação } \\
\text { - "saber sobre" (knowing about) } \\
\text { • Conhecimento declarativo }\end{array}$ & $\begin{array}{l}\text { - "saber como" (know how, } \\
\text { knowing how) } \\
\text { • Conhecimento procedural }\end{array}$ \\
\hline Formas & $\begin{array}{l}\text { - Cognitivo } \\
\text { - Incorporado a habilidades } \\
\text { - Incorporado a produtos e serviços }\end{array}$ & $\begin{array}{l}\text { - Cognitivo } \\
\text { - Incorporado a habilidades } \\
\text { - Incorporado a produtos e serviços } \\
\text { - Emotivo } \\
\text { - Sócio-cultural } \\
\text { - Semântico } \\
\text { - Sagaz }\end{array}$ \\
\hline Componentes & & $\begin{array}{l}\text { - Consciente } \\
\text { - Automático } \\
\text { - Coletivo }\end{array}$ \\
\hline
\end{tabular}

Figura 1- Classificações do conhecimento organizacional 
mação. Essa dimensão é definida por esses autores apenas através de exemplos, citando o número de operações que uma tarefa exige em uma CPU para ser completada. Grant (1996) define outras três dimensões: transferibilidade, o grau com que o conhecimento pode ser transferido sem degradação; agregabilidade, i.e., a capacidade de diferentes elementos do conhecimento serem combinados; e apropriabilidade, o grau em que o indivíduo é capaz de receber retorno de igual valor ao conhecimento que possui. Embora de uma corrente de pesquisa diversa das focalizadas aqui, Chai et al. (2003) apontam para dependência do contexto (tradução do termo em inglês embeddedness) como dimensão, ou construto, importante no estudo do conhecimento. O quadro da Figura 2 organiza as dimensões de análise para o conhecimento organizacional propostas.

Outra distinção existente é relativa aos diferentes níveis de análise utilizados nos estudos sobre conhecimento organizacional. A literatura faz uso principalmente de três: o individual, o de grupo e o organizacional (CROSSAN et al., 1999). Desses, alguns trabalhos fazem uso de dois: o individual e o organizacional (e.g. CONNNER e PRAHALAD, 1996; COHEN e LEVINTHAL, 1990). Outros focalizam especificamente o nível dos grupos (e.g. LEONARD e SENSIPER, 1998), enquanto outros utilizam os três níveis de análise (e.g. CROSSAN et al., 1999; FLEURY e FLEURY, 2000). Um quarto nível, o inter-organizacional, é utilizado em menor grau por alguns autores (e.g. HEDLUND, 1994). Porém, o crescente interesse por assuntos inter-organizacionais, como redes entre empresas, cadeias produtivas e arranjos produtivos locais, deve impulsionar as discussões neste último

\begin{tabular}{ll}
\hline \multicolumn{1}{c}{ Dimensão } & \multicolumn{1}{c}{ Descrição } \\
\hline Codificabilidade & $\begin{array}{l}\text { Grau no qual o conhecimento pode } \\
\text { ser transformado em um conjunto de } \\
\text { regras e relações }\end{array}$ \\
Complexidade & $\begin{array}{l}\text { Definido indiretamente pelo esforço } \\
\text { necessário para codificar o conhecimento }\end{array}$ \\
Transferibilidade & $\begin{array}{l}\text { Grau em que o conhecimento pode } \\
\text { ser transferido sem degradação }\end{array}$ \\
Agregabilidade & $\begin{array}{l}\text { Grau com que o conhecimento } \\
\text { pode ser combinado }\end{array}$ \\
Apropriabilidade & $\begin{array}{l}\text { Grau pelo qual o detentor do } \\
\text { conhecimento pode obter retorno }\end{array}$ \\
$\begin{array}{l}\text { Dependência } \\
\text { do contexto }\end{array}$ & $\begin{array}{l}\text { Grau em que o conhecimento é } \\
\text { contexto sócio-cultural, político, } \\
\text { econômico e financeiro }\end{array}$ \\
\hline
\end{tabular}

Figura 2 - Dimensões do conhecimento organizacional nível de análise (para uma discussão conceitual sobre gestão do conhecimento em redes de organizações, ver NAKANO, 2005). Em resumo, o uso de diferentes níveis de análise é uma das características da literatura sobre conhecimento, e em particular, daquela referente à Aprendizagem Organizacional (CROSSAN et al., 1999).

\section{Conhecimento na literatura em organizações: perspectivas}

Duas perspectivas diferentes quanto à natureza do conhecimento organizacional estão presentes na literatura (VON KROGH et al., 1994, CHAI et al., 2003). Na perspectiva tradicional, ele é visto como abstrato, com finalidade específica e orientado para a resolução de problemas. É chamada de perspectiva cognitiva, que vê o mundo como uma realidade prédefinida, de existência independente da ação e história da organização e dos indivíduos (esta perspectiva é chamada por alguns teóricos organizacionais de perspectiva positivista). Em contraste, existe a perspectiva autopoiética (do grego auto $=$ próprio, poiein $=$ fazer, produzir, conceber), também chamada construtivista (VON KROGH, 1998). Nela a realidade não é um estado pré-definido, pelo contrário, é construída através das observações e reflexões do indivíduo, em sua interação com a situação existente (ou, em Teoria das Organizações, perspectiva interpretativa).

Na perspectiva cognitiva, a tarefa do indivíduo ou organização é produzir a representação mais acurada da realidade, através da coleta e interpretação de informações, a fim de tomar decisões acertadas. Organizações são sistemas cuja finalidade é o processamento de informações e manipulação de símbolos. A aprendizagem nesse caso se dá fundamentalmente pela obtenção de representações cada vez mais precisas da realidade, principalmente através de informações oriundas de novas experiências (learning by doing) (LEVITT e MARCH, 1988). Apesar de críticas a esta perspectiva, que partem do argumento que o conhecimento não pode ser desvinculado de seu caráter subjetivo e de sua dependência do contexto (THOMPSON e WALSHAM, 2004), ele é utilizado por diversos autores.

Um exemplo é o trabalho de Conner e Prahalad (1996). Esses autores entendem o conhecimento como uma possessão dos indivíduos, utilizando a expressão "estoque de conhecimento". O estoque é composto de experiência, insights e habilidades adquiridos através da interpretação dos resultados das ações que a organização tomou. Da mesma forma, Cohen e Levinthal (1990) definem o conhecimento como as habilidades, linguagem e informações que a organização adquire ao interagir com o ambiente. Ambos os trabalhos compartilham a noção de conhecimento como estado ou condição, dos indivíduos e das organizações a que eles 
pertencem, resultado da interpretação das respostas do ambiente às suas ações.

A perspectiva construtivista (VON KROGH, 1998) vê o conhecimento como um dos componentes do processo de compreensão do mundo. A cognição deixa de ser a descoberta de relações pré-existentes entre fatos, eventos e fenômenos, como dentro da perspectiva cognitiva, e passa a ser o "ato criativo de conceber (gerar) o mundo" (VON KROGH et al., 1994, p.58, traduzido pelo autor). $\mathrm{O}$ conhecimento deixa de ser intimamente ligado à informação, e passa a ser ligado mais fortemente à observação. Por sua vez, a informação deixa de ser um ente objetivo, uma propriedade, e passa a ser processo de interpretação da realidade.

A proposição de von Krogh e colegas (VON KROGH et al.,1994; VON KROGH, 1998) é baseada em trabalhos sobre Neurobiologia (principalmente de Maturana e Varela), mas é clara a similaridade da perspectiva construtivista com o trabalho sobre sociologia do conhecimento de Berger e Luckmann (1967). Ambas enfatizam o conhecimento como gerado em um processo de construção, resultado de interações entre o indivíduo e o meio que o circunda e entre indivíduos e grupos. O conhecimento não é uma realidade pré-existente, mas é socialmente construído. Sob essa perspectiva, organizações são locais, físicos ou virtuais, onde acontecem interações (NONAKA e KONNO, 1998), e conhecimento é gerado e transferido através de processos primariamente sociais (e.g. HEDLUND, 1994; NONAKA, 1994). Em contraste, a perspectiva cognitiva vê organizações como sistemas de processamento de informações, com ênfase no fluxo e interpretação das mesmas. Conhecimento é gerado através do processo de interpretação das informações provenientes do ambiente e de experiências passadas (e.g. MARCH e OLSEN, 1979; DAFT e WEICK, 1984).

Em resumo, existem duas perspectivas quanto à natureza do conhecimento organizacional. Tomando emprestada a terminologia de von Krogh (1998), essas correntes são: a cognitiva e a construtivista. O quadro da Figura 3 resume as principais características de cada uma delas.

\section{Processos de aprendizagem e Gestão do Conhecimento Organizacional}

Uma das preocupações centrais dos estudiosos do conhecimento organizacional é referente aos processos utilizados pelas organizações para aprender e administrar o que sabem. Fleury e Fleury (2000) por exemplo, destacam três processos principais: a aquisição e desenvolvimento de conhecimentos, sua disseminação e a construção da memória organizacional. Dado o interesse sobre os processos de aprendizagem e gestão do conhecimento, existem na literatura diversas proposições sobre o assunto, e em trabalho anterior (NAKANO e FLEURY, 2001) foram selecionadas e analisadas doze delas. Retoma-se e amplia-se aqui a análise iniciada naquele trabalho, com o objetivo de sintetizar os principais processos de aprendizagem e gestão do conhecimento propostos na literatura. Não são repetidas aqui as análises já realizadas, por exemplo quanto à classificação das proposições entre as diferentes correntes de pesquisa. $\mathrm{O}$ quadro da Figura 4 apresenta as proposições analisadas.

A comparação das doze proposições revela duas dimensões diferenciadoras: a primeira referente ao seu caráter, se prescritivo, i.e., com o objetivo de preconizar ações e princípios para a modificação da organização; ou se analítico, i.e., voltada para a elaboração de conceitos e construtos que permitam a descrição, análise e compreensão do fenômeno. Por exemplo, a proposta de Senge (1990) preconiza princípios, ou disciplinas como o autor denomina, para a criação de uma organização de aprendizagem, assim como Watkins e Marsick (1993), que propõem um conjunto de ações para o mesmo fim. Ambas compartilham o pressuposto da existência de um tipo ideal de organização, a ser atingido pelo uso dos princípios ou ações por elas prescritas. Por outro lado, proposições como as de March e Olsen (1979), Daft e Weick (1984) e Huber (1991) são analíticas, têm por objetivo definir o que é a aprendizagem organizacional, e principalmente, compreender como ela se processa.

\begin{tabular}{|c|c|c|}
\hline & Perspectiva Cognitiva & Perspectiva Construtivista \\
\hline $\begin{array}{l}\text { Conhecimento } \\
\text { Organizacional }\end{array}$ & $\begin{array}{l}\text { É o resultado do processo de interpretação de } \\
\text { informações do ambiente. Tem finalidade específica } \\
\text { e é orientado à resolução de problemas }\end{array}$ & $\begin{array}{l}\text { É um componente do processo de definição de } \\
\text { problemas e construção da realidade, e não seu } \\
\text { resultado final. É dependente do contexto e da história } \\
\text { prévia da organização e dos indivíduos }\end{array}$ \\
\hline Informação & $\begin{array}{l}\text { É a "entrada", a "matéria-prima" através da qual a } \\
\text { organização elabora interpretações, gera conhecimento } \\
\text { e toma decisões }\end{array}$ & $\begin{array}{l}\text { É o próprio processo de interpretação, através do qual } \\
\text { o conhecimento é adquirido }\end{array}$ \\
\hline Organização & É um sistema de processamento de informações & $\begin{array}{l}\text { É o local onde se realizam interações, através das quais } \\
\text { o conhecimento é gerado, transformado e transferido }\end{array}$ \\
\hline
\end{tabular}

Figura 3 - Perspectivas relativas ao conhecimento organizacional 


\begin{tabular}{|c|c|c|}
\hline Proposição & Corrente de pesquisa & Processos Sugeridos \\
\hline $\begin{array}{c}\text { March e } \\
\text { Olsen (1979) }\end{array}$ & $\begin{array}{l}\text { Aprendizagem } \\
\text { Organizacional }\end{array}$ & $\begin{array}{l}\text { - Ação individual } \\
\text { - Ação Organizacional } \\
\text { - Resposta do ambiente } \\
\text { - Crenças individuais }\end{array}$ \\
\hline $\begin{array}{c}\text { Daft e } \\
\text { Weick (1984) }\end{array}$ & $\begin{array}{l}\text { Aprendizagem } \\
\text { Organizacional }\end{array}$ & $\begin{array}{l}\text { - Prospecção } \\
\text { - Interpretação } \\
\text { - Aprendizagem }\end{array}$ \\
\hline Huber (1991) & $\begin{array}{l}\text { Aprendizagem } \\
\text { Organizacional }\end{array}$ & $\begin{array}{l}\text { - Aquisição de conhecimento } \\
\text { - Distribuição de informação } \\
\text { - Interpretação de informação } \\
\text { - Memória organizacional }\end{array}$ \\
\hline March (1991) & $\begin{array}{l}\text { Aprendizagem } \\
\text { Organizacional }\end{array}$ & $\begin{array}{l}\text { - Descoberta } \\
\text { - Exploração }\end{array}$ \\
\hline $\begin{array}{l}\text { Crossan } \\
\text { et al. (1999) }\end{array}$ & $\begin{array}{l}\text { Aprendizagem } \\
\text { Organizacional }\end{array}$ & $\begin{array}{l}\text { - Intuição } \\
\text { - Interpretação } \\
\text { - Integração } \\
\text { - Institucionalização }\end{array}$ \\
\hline Senge (1990) & $\begin{array}{c}\text { Organizações } \\
\text { de Aprendizagem }\end{array}$ & $\begin{array}{l}\text { - Domínio pessoal } \\
\text { - Modelos mentais } \\
\text { - Visão compartilhada } \\
\text { - Aprendizado em grupo } \\
\text { - Pensamento sistêmico }\end{array}$ \\
\hline $\begin{array}{c}\text { Watkins e } \\
\text { Marsick (1993) }\end{array}$ & $\begin{array}{c}\text { Organizações } \\
\text { de Aprendizagem }\end{array}$ & $\begin{array}{l}\text { - Criar oportunidades de aprendizagem contínua } \\
\text { - Promover questionamento e diálogo } \\
\text { - Encorajar colaboração e aprendizado em grupo } \\
\text { - Estabelecer sistemas para capturar e compartilhar o aprendizado } \\
\text { - Dar poder às pessoas em direção à uma visão comum }\end{array}$ \\
\hline Hedlund (1994) & $\begin{array}{c}\text { Gestão do } \\
\text { Conhecimento }\end{array}$ & $\begin{array}{l}\text { - Articulação } \\
\text { - Internalização } \\
\text { - Extensão } \\
\text { - Apropriação } \\
\text { - Assimilação }\end{array}$ \\
\hline $\begin{array}{c}\text { Nonaka e } \\
\text { Takeuchi (1995) }\end{array}$ & $\begin{array}{c}\text { Gestão do } \\
\text { Conhecimento }\end{array}$ & $\begin{array}{l}\text { - Socialização } \\
\text { - Externalização } \\
\text { - Combinação } \\
\text { - Internalização }\end{array}$ \\
\hline Hansen et al. (1999) & $\begin{array}{l}\text { Gestão do } \\
\text { Conhecimento }\end{array}$ & $\begin{array}{l}\text { - Codificação } \\
\text { - Personalização }\end{array}$ \\
\hline Ruggles (1999) & $\begin{array}{l}\text { Gestão do } \\
\text { Conhecimento }\end{array}$ & $\begin{array}{l}\text { - Gerar novo conhecimento } \\
\text { - Acessar conhecimento valioso } \\
\text { - Usar conhecimento acessível } \\
\text { - Representar o conhecimento em documentos e bases de dados } \\
\text { - Facilitar o conhecimento através da cultura }\end{array}$ \\
\hline $\operatorname{Tan}(2000)$ & $\begin{array}{c}\text { Gestão do } \\
\text { Conhecimento }\end{array}$ & $\begin{array}{l}\text { - Identificação de ativos de conhecimento } \\
\text { - Criação de conhecimento } \\
\text { - Encapsulação do conhecimento } \\
\text { - Compartilhamento e disseminação do conhecimento } \\
\text { - Recuperação do conhecimento }\end{array}$ \\
\hline
\end{tabular}

Figura 4 - Proposições analisadas

Uma segunda dimensão diferenciadora entre as proposições é referente à sua perspectiva, se cognitiva, i.e., voltada para a aquisição e interpretação da informação; ou se construtivista, i.e., que prioriza os processos sociais de tradução e transformação do conhecimento. Por exemplo, as proposições de Ruggles (1999) e Tan
(2000), embora tenham caráter prescritivo como as de Senge (1990) e Watkins e Marsick (1993), diferenciamse pelo seu foco mais restrito, sobre o fluxo de informações dentro da organização, e pelo fato de não prescreverem ações de modificação na área comportamental. Da mesma forma, as propostas de Crossan et al. (1999), 
Hedlund (1994) e Nonaka e Takeuchi (1995) se diferenciam das outras de caráter analítico, como as de Huber (1991), March e Olsen (1976) e Daft e Weick (1984), por enfatizarem a transferência e transformação do conhecimento, do âmbito pessoal para o organizacional e do caráter tácito para o explícito. O quadro da Figura 5 apresenta as doze proposições que compõem o conjunto em pauta, divididas segundo essas dimensões.

\begin{tabular}{lll}
\hline & Caráter Prescritivo & Caráter Analítico \\
\hline $\begin{array}{lll}\text { Perspectiva } \\
\text { Cognitiva }\end{array}$ & Tan (2000) & March e Olsen (1979) \\
& & Daft e Weick (1984) \\
& Huber (1991) \\
& & March (1991) \\
& & Hansen et al. (1999) \\
& & Ruggles (1999) \\
\hline Perspectiva & Senge (1990) & Crossan et al. (1999) \\
Construtivista & Watkins e Marsick (1993) & Hedlund (1994) \\
& & Nonaka e Takeuchi (1995) \\
& & \\
\hline
\end{tabular}

Figura 5 - Proposições classificadas segundo as dimensões propostas

As proposições de caráter prescritivo, como por exemplo as de Senge (1990) e Watkins e Marsick (1993), são fortemente focalizadas na implementação de mudanças na organização. Tendem a ser mais ecléticas, valendo-se, para a seleção de suas recomendações, mais do critério da aplicabilidade dos conceitos do que do seu rigor conceitual e metodológico (EASTERBYSMITH, 1997). Por esses motivos, sem desmerecer seu valor, serão analisadas em mais detalhe somente as proposições de caráter analítico, já que um dos objetivos deste texto é a montagem de um modelo de referência para o estudo de campo a ser apresentado.

\subsection{Análise das proposições de caráter analítico}

O exame das proposições de caráter analítico revela que a perspectiva adotada em cada proposição, se cognitiva ou construtivista, determina diferenças nos processos por elas preconizados. As proposições com perspectiva cognitiva focalizam o fluxo do conhecimento explícito nas organizações, enquanto as de perspectiva construtivista enfatizam os processos de transformação do conhecimento internos à organização. Uma perspectiva privilegia o fluxo e a interpretação de informações, enquanto a outra, a transformação do conhecimento. Corroborando a tese de von Krogh e Roos (1994), entende-se que a distinção é de cunho epistemológico: para o primeiro conjunto de proposi- ções a realidade é objetiva e pré-existente, e cabe à organização colher informações e interpretá-las. Em contraste, para o segundo, o conhecimento é primordialmente criado no interior de cada indivíduo: o foco recai sobre o conhecimento tácito, e a organização deve preocupar-se em criar formas de torná-lo disponível a todos os seus membros. A seguir, estuda-se cada conjunto de proposições.

\subsection{A perspectiva construtivista}

As propostas de perspectiva construtivista (CROSSAN et al., 1999; HEDLUND, 1994; NONAKA e

TAKEUCHI, 1995) têm características mais homogêneas. De forma geral, eles estudam dois fenômenos: a conversão do conhecimento, de tácito em explícito e vice-versa, e a transformação do conhecimento entre os níveis individual, de grupo e organizacional. Esses fenômenos não são independentes entre si, e por esse motivo, os seus modelos são cíclicos e com etapas recursivas. O quadro da Figura 6 coloca lado a lado as proposições dos três trabalhos e os processos que preconizam.

O quadro da Figura 6 mostra que os temas recorrentes nas propostas são: (1) a tradução do conhecimento tácito individual para o explícito organizacional e (2) a conversão do conhecimento organizacional em tácito individual, através de processos sociais. Também é ressaltada a importância do indivíduo e sua óptica particular na criação e absorção de novo conhecimento.

A comparação também revela ênfases de cada proposta: Crossan et al. (1999) detalham o processo de institucionalização do conhecimento, idéia que embora presente em Nonaka e Takeuchi (1995) no processo de combinação, é mais claramente articulada pelos primeiros autores: é na institucionalização que o conhecimento individual é incorporado a estruturas, sistemas e procedimentos organizacionais. Por sua vez, Nonaka e Takeuchi (1995) são os únicos a destacar o processo de transferência de conhecimento tácito entre indivíduos (socialização), passo fundamental no seu entender para sua posterior tradução em explícito, através da interação de vários indivíduos. Já Hedlund (1994) se diferencia por integrar o ambiente em seu modelo, destacando os processos pelos quais há fluxos de conhecimento de e para o exterior da organização, aos quais chama de assimilação e disseminação.

\subsection{A perspectiva cognitiva}

A análise das propostas de perspectiva cognitiva (MARCH e OLSEN, 1979; DAFT e WEICK, 1984; HUBER, 1991; MARCH, 1991; HANSEN et al., 1999; 


\begin{tabular}{|c|c|c|c|}
\hline Processo & Crossan et al. (1999) & Hedlund (1994) & Nonaka e Takeuchi (1995) \\
\hline Aquisição de conhecimento & $\begin{array}{c}\text { Intuição (especificamente } \\
\text { conhecimento tácito individual) }\end{array}$ & Assimilação & Não discutido \\
\hline $\begin{array}{l}\text { Transferência de conhecimento } \\
\text { tácito entre indivíduos }\end{array}$ & Não discutido & Não discutido & Socialização \\
\hline $\begin{array}{l}\text { Tradução do conhecimento } \\
\text { tácito individual para explícito }\end{array}$ & Interpretação e Integração & Articulação & Externalização \\
\hline $\begin{array}{l}\text { Elaboração e construção de } \\
\text { novo conhecimento explícito }\end{array}$ & Integração & Não discutido & Combinação \\
\hline $\begin{array}{l}\text { Formalização do conhecimento } \\
\text { em procedimentos e rotinas }\end{array}$ & Institucionalização & Extensão & Combinação \\
\hline $\begin{array}{l}\text { Aquisição de novo } \\
\text { conhecimento tácito individual } \\
\text { a partir do explícito organizacional }\end{array}$ & Intuição & Internalização & Internalização \\
\hline Distribuição do conhecimento & $\begin{array}{l}\text { Implicitamente assumido no } \\
\text { processo de Institucionalização }\end{array}$ & Disseminação & $\begin{array}{l}\text { Implicitamente assumido no } \\
\text { processo de socialização }\end{array}$ \\
\hline
\end{tabular}

Figura 6 - Proposições de Perspectiva Construtivista

RUGGLES, 1999) revela maior diversidade que o conjunto anterior. Alguns trabalhos focalizam mais fortemente determinados processos, como Daft e Weick (1984), que detalham aquisição e interpretação da informação, e March e Olsen (1979) que além desses processos, discutem a geração de conhecimento. Já outros trabalhos se atêm a características do uso e conservação do conhecimento e a tensão entre a criação e descoberta do novo e a exploração e uso do existente, como March (1991), ou analisam diferentes formas de armazenamento, como Hansen et al. (1999).

Os principais processos analisados por essas proposições são: (a) criação e aquisição, (b) interpretação, (c) armazenamento (também chamado de memória organizacional) e (d) disseminação. Criação e aquisição são processos semelhantes, porém o primeiro se refere à geração de novo conhecimento como uma atividade interna à organização, enquanto o segundo é o processo de importar novo conhecimento de fontes externas. Interpretação é o processo de compreensão e atribuição de significado às informações adquiridas, um processo realizado por indivíduos (LEVITT e MARCH, 1988), através de processos cognitivos (DODGSON, 1993). Armazenamento, ou memória, engloba a estocagem de informação bem como os processos relativos a ela: alimentação de novas informações, manutenção em estoque e sua recuperação. A disseminação se refere à sua distribuição, através da qual as informações são feitas disponíveis para toda a organização (HUBER, 1991; LEVITT e MARCH, 1988; WALSHe UNGSON,
1991). Os processos são representados na Figura 7.
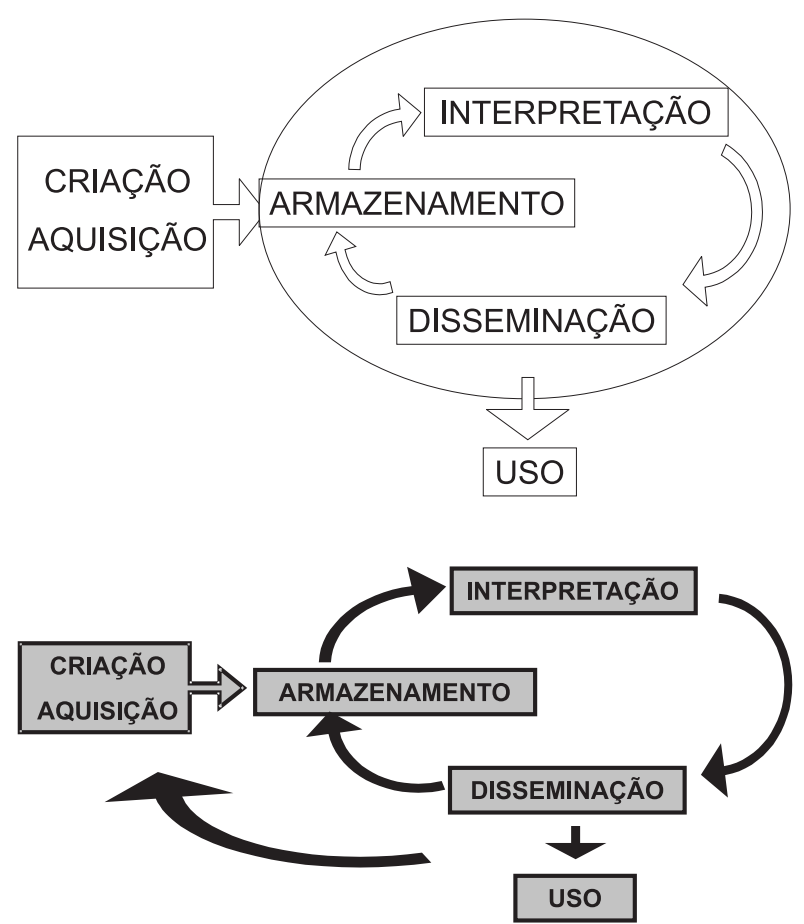

Fonte: os autores

Figura 7 - Processos de Aprendizagem e Gestão do Conhecimento das Proposições de perspectiva Cognitiva

A Figura 7 indica que os processos de interpretação, armazenamento e disseminação são cíclicos, não 
obedecem uma ordem pré-estabelecida: conhecimento pode ser interpretado antes de ser armazenado e disseminado, interpretado para então ser armazenado, etc. Outro ponto a se observar é que o conhecimento é inexaurível, i.e., pode ser repetidas vezes retirado do estoque e utilizado, sem que sofra desgaste ou depreciação (por exemplo um procedimento padrão é executado repetidas vezes, e o conhecimento nele incorporado não sofre desgaste). Pelo contrário, a literatura sugere que o conhecimento se desgasta e se perde quando não utilizado (ARGOTE et al., 1990; DARR et al., 1995). O conhecimento estocado em uma organização é ao mesmo tempo inexaurível e perecível, pode ser utilizado repetidas vezes, porém é sujeito à perdas e depreciação.

Quanto à disseminação, deve-se lembrar que "organizações freqüentemente não sabem o que sabem" (HUBER, 1991, p. 100, traduzido pelo autor). A disseminação do conhecimento tem papel central na aprendizagem organizacional: muitas organizações não sabem o que têm armazenado, e por esse motivo deixam de tirar vantagem daquilo que já possuem (O’DELL e GRAYSON, 1998). As deficiências provenientes da fraca comunicação dentro da organização são extensamente discutidas, por exemplo, na literatura sobre desenvolvimento de novos produtos (e.g. CARTER e BAKER, 1992). Essa literatura aponta a "compartimentalização" proveniente da estrutura burocrática como causadora de deficiente disseminação do conhecimento, e propõe formas alternativas de organização, como a Engenharia Simultânea, para resolver esse problema (KRUGLIANKAS, 1993).

Outras formas de disseminação do conhecimento são: os processos de socialização, quando novos membros são aculturados na organização (LEVITT e MARCH, 1988), o uso de procedimentos padronizados (MARCH e SIMON, 1958), as práticas de RH como treinamentos, visitas e rotação de funções (GARVIN, 1994; NONAKA e KONNO, 1998), os grupos de discussão (O'DELL e GRAYSON ,1998), as forças-tarefa para a resolução de problemas específicos (NONAKA e KONNO, 1998), os grupos de especialistas que oferecem suporte aos membros da organização (MOORE e BIRKINSHAW, 2000), as listas de discussão eletrônicas (O'DELL e GRAYSON, 1998; OLIVERA, 2000), e o uso de intranets e softwares de comunicação (CHAIT, 1999; NONAKA e KONNO, 1998; OLIVERA, 2000).

A figura também sugere que o ciclo armazenamento interpretação - disseminação - uso facilita a criação e aquisição de novos conhecimentos, de acordo com proposições como a de capacidade de absorção (COHEN e LEVINTHAL, 1990).

\section{Conclusão}

O conhecimento organizacional e sua gestão são temas complexos e multi-facetados. Como comentam Fleury e Oliveira Jr. (2001) “O tema Gestão do Conhecimento emergiu, na última década, das discussões fechadas entre pesquisadores da área de Ciência e Tecnologia e profissionais de $\mathrm{P} \& \mathrm{D}$ (Pesquisa e Desenvolvimento) das empresas, para despontar como um dos assuntos mais polêmicos e menos compreendidos." (p.15). Adicionalmente, no campo estritamente acadêmico, as discussões provêm de diversas origens e é possível encontrar diversas proposições. Este artigo procurou compilar, organizar e analisar algumas das principais contribuições na área.

A análise de literatura conduzida neste artigo indicou que os autores utilizam diferentes classificações, dimensões e níveis de análise para estudar o conhecimento, e partem de diferentes enfoques epistemológicos, o que possibilita dividir a literatura em duas perspectivas básicas: a construtivista e a cognitiva. A perspectiva cognitiva vê o conhecimento como um insumo, que embora intangível e complexo, é passível de ser armazenado, combinado e reutilizado. A gestão do conhecimento nesta perspectiva aproxima-se da gestão do processo produtivo de um bem intangível: são utilizados conceitos e palavras como criação, armazenamento, disseminação e utilização. Alguns dos problemas na gestão desse processo são: o caráter dinâmico do conhecimento, sua volatilidade e a diversidade de formas de armazenamento possíveis. Assim, dentro desta perspectiva, gestão do conhecimento é a administração eficaz das atividades relativas à aquisição ou criação, a utilização e a manutenção de um ativo intangível.

Já na perspectiva construtivista, o conhecimento ganha um aspecto mais complexo: ele não é apenas um bem intangível a ser mantido e utilizado, ele é o próprio processo de criação, e é o resultado da interação entre as pessoas. $\mathrm{O}$ conhecimento é construído no relacionamento entre indivíduos, é dependente de suas capacidades e de suas características pessoais, do contexto e das formas de expressão escolhidas. É subjetivo, fugidio. Na gestão do conhecimento, de acordo com esta perspectiva, ganha destaque a administração das pessoas, a necessidade da oferta de possibilidades de interação, a comunicação interpessoal. O tácito ganha papel central, porém entende-se que ele é traduzível e administrável. Neste ponto existe uma contradição apontada por alguns (SCHULTZE e STABELL, 2004), principalmente no modelo de Nonaka e Takeuchi (1995), o mais conhecido dentre estes: embora se reconheça que o conhecimento tácito é o importante, é preciso traduzi-lo para explícito, de forma a poder administrá-lo.

Também pode-se diferenciar os trabalhos pelo seu caráter, se prescritivo ou analítico. As críticas quanto a essas duas abordagens são as conhecidas: as análises analíticas se mais interessantes e que possibilitam compreensão mais profunda do tema, são por outro lado vagas e de difícil aplicação prática (embora possa se argumentar 
que esse não é o seu foco). Já as proposições prescritivas, que partem do pressuposto que há uma forma ótima de gerir o conhecimento, que deveria ser seguido por todas as organizações, pecam pelo determinismo e pelo reducionismo. A visão educada sobre conhecimento organizacional e sua gestão deveria ser capaz de conseguir equilíbrio entre as duas abordagens.

Uma limitação importante deste artigo é que ele deixa de lado análises que de certa forma não estão alinhadas com as discussões mais freqüentes na literatura (o chamado mainstream), como no caso do trabalho de Schultze e Stabell (2004). Esses autores criticam especialmente o que aqui foi denominado perspectiva cognitiva, a partir do argumento de que o conhecimento é por demais complexo e ambíguo para poder ser analisado de forma independente do seu contexto (e, conforme comentado acima, também a perspectiva construtivista). Porém, como seria por demais pretensioso pretender esgotar assunto tão vasto como o conhecimento, entende-se que esta é uma limitação aceitável. É evidente também que esta análise, embora pretenda ser abrangente, não cobre a totalidade de proposições e modelos de gestão do conhecimento (e.g. LYTRAS et al., 2002). Futuros trabalhos que continuem a discussão aqui iniciada são mais do que necessários.

Espera-se que a análise aqui oferecida preencha uma lacuna na literatura em língua portuguesa, e que contribua para a aquisição de uma visão educada sobre conhecimento, que auxilie não somente o avanço do seu estudo, mas sua melhor gestão dentro das organizações, ação fundamental para a manutenção e melhoria de sua competitividade.

\section{Referências}

ADLER, P. S. e COLE, R. E. Designed for learning: A tale of two autoplants. Sloan Management Review, spring 1993, p.85-94

ARGOTE, L.; BECKMAN, S. L.; EPPLE, D. The persistence and transfer of learning in industrial settings. Management Science, v.36. n.2, February 1990, p.140-154

ARGYRIS, C.; SCHON, D. A. Organizational Learning. Reading, MA: Addison-Wesley, 1978

BERGER, P.; LUCKMANN, T. The Social Construction of Reality. New York: Anchor Books, 1967, 219p.

BERGGREN, C. NUMMI vs. Uddevalla. Sloan Management Review, winter, 94, p.37-49

BONTIS, N.; CROSSAN, M.; HULLAND, J. Managing an organizational learning system by aligning stocks and flows. Journal of Management Studies, v. 39, n. 4, June 2002.

CARTER, D. E.; BAKER, B. S. Concurrent Engineering, the Product Development Enviroment for the 1990s. Addison-Wesley, 1992.

CASTILLO, J. A note on the concept of Tacit knowledge. Journal of Management Inquiry, v. 11, n. 1, Mar 2002.

CHAI, K-H.; GREGORY, M.; SHI, Y. Bridging islands of knowledge: a framework of knowledge sharing mechanisms. International Journal of Technology Management, v.25, n.8, p.703-727, 2003.

CHAIT, L. P. Creating a successful Knowledge Management System. Journal of Business Strategy, March/April 1999, p. 23-26.

COHEN, W. M.; LEVINTHAL, D. A. Absortive capacity: A new perspective on learning and innovation. Administrative Science Quarterly, v.35, 1990, p.128-52.

CONNER, K.; PRAHALAD, C. K. A Resource-based theory of the firm: knowledge versus opportunism. Organization Science, v.7, n.5, Sep-Oct 1996, p.477501.

CROSSAN, M. M.; LANE, H. W.; WHITE, R. E. An organizational learning framework: from intuition to institution. The Academy of Management Review, v.24, n.3, Jul 1999, p.522-37.

CROSSAN, M. M.; LANE, H. W.; WHITE, R. E.; DJURFELDT, L. Organizational learning: dimensions for a theory. The International Journal of Organizational Analysis, v.3, n.4, October 1995, p.337-60.

CYERT, R. M.; KUMAR, P. Technology management and the future. IEEE Transactions on Engineering Management, v.41, n.4, November, 1994, p.333-4.

DAFT, R. L.; WEICK, K. E. Toward a model of organizations as interpretative systems. The Academy of Management Review, v.9, n.2, 1984, p.284-95.

DARR, E. D.; ARGOTE, L.; EPPLE, D. The acquisition, transfer, and depreciation of knowledge in service organizations: productivity in franchises. Management Science, v.41, n.11, November 1995, p.17501762.

DODGSON, M. Organizational learning: a review of some literatures. Organization Studies, v.14, n.3, 1993. 
EASTERBY-SMITH, M. Disciplines of organizational learning: contributions and critiques. Human Relations, v.50, n.9, 1997, p. 1085-1113.

EASTERBY-SMITH, M.; CROSSAN, M.; NICOLINI, D. Organizational learning: debates past, present and future. Journal of Management Studies, v.37, n.6, Sept 2000, p. 783-96.

EASTERBY-SMITH,M.;SNELL, R.; GHERARDI, S. Organizational learning: diverging communities of practice? Management Learning, v.29, n.3, 1998, p.259-272.

FIOL, C. M.; LYLES, M.A. Organizational learning. The Academy of Management Review, v.10, n.4, Oct. 1985, p.803-14.

FLEURY,A.C.C.eFLEURY,M. T.L.Aprendizageme Inovação Organizacional. São Paulo: Atlas, 1995

FLEURY,A. C. C. e FLEURY, M. T.L. Estratégias Empresariais e Formação de Competências. São Paulo: Atlas, 2000.

FLEURY,M.T.L. e OLIVEIRA Jr., M. M. (org.) Gestão Estratégica do Conhecimento. São Paulo: Atlas, 2001.

GARVIN, D. A. Building a learning organization. Harvard Business Review, Jul-Aug 93, p.78-91.

GRANT, R. M. Toward a knowledge-based theory of the firm. Strategic Management Journal, v.17, Winter Special Edition, 1996, p. 109-22.

HANSEN, M. T.; NOHRIA, N.; TIERNEY, T. What's your strategy for managing knowledge? Harvard Business Review, v.77, n.2, March-April 1999. p.106-16.

HEDBERG, Bo. How organizations learn and unlearn In: NYSTROM, Paul C. and STARBUCK, William H.(ed.) Handbook of Organizational Design. Oxford: Oxford University Press, 1981.

HEDLUND, G. A model of knowledge management and the $\mathrm{N}$-form corporation. Strategic Management Journal, v.15, 1994, p.73-90.

HIRSCH, P. M.; LEVIN, D. Z. Umbrella advocates versus Validity policy: A life-cycle model. Organization Science, v.10, n.2, March/April 1999, p. 199-212.

HUBER, G. P. Organizational learning: the contributing processes and the literatures. Organization Science, v.2, n.1, Feb. 1991, p.88-115.

KAHANER, L. Competitive Intelligence. New York: Touchstone, 1996.
KYRIAKOPOLOUS, K.; RUYTER, K. Knowledge Stocks and Information Flows in New Product Development. Journal of Management Studies, v. 41, n. 4, p. 1469-1498, 2004.

KOGUT, B.; ZANDER, U. Knowledge of the firm: combinative capabilities and the replication of technology. Organization Science, v.3, n.3, Aug 1992, p.383-97.

KRUGLIANSKAS, I. Engenharia simultânea: organização e implantação em empresas brasileiras. Revista de Administração da USP, São Paulo, v. 28, nº 4, p.104110, out/dez. 1993.

LAM, A. Organizational Learning in Multinationals: R\&D Networks of Japanese and US MNEs in the UK. Journal of Management Studies, v. 40.n.3, p.673-703, 2003.

LEONARD, D.; SENSIPER, S. The role of tacit knowledge in group innovation. California Management Review, v.40, n.3, 1998, p.112-132.

LEVITT, B.; MARCH, J. G. Organizational learning. Annual Review of Sociology, v.14, 1988, p.319-340.

LYTRAS, M.D., POULOUDI, N. and POULYMENAKOU, A. Knowledge management convergence: expanding learning frontiers, Journal of Knowledge Management, v. 6, n. 1, p.40-51, 2002.

MARCH, J. G. Exploration and exploitation in organizational learning. Organization Science, v.2, n.1, Feb. 1991, p.71-87.

MARCH, J. G.; OLSEN, J. P. Organizational learning and the ambiguity of the past. In: MARCH, J. G. and OLSEN, J. P. (ed.) Ambiguity and Choice in Organizations. Bergen, Norway: Universitetsforlaget, 1979 ( $\left.2^{\text {nd }} \mathrm{ed}\right), 408 \mathrm{p}$.

MARCH, J. G.; SIMON, H. A. Organizations. New York: John Wiley, 1958, 262p.

MINER, A. S.; MEZIAS, S. J. Ugly duckling no more: past and futures of organizational learning. Organization Science, v.7, n.1, Jan-Feb 1996, p.88-98.

MOORE, K.; BIRKINSHAW, J. Managing knowledge in global service firms: centers of excellence. The Academy of Management Executive, v.12, n.4, 1998, p.81-92.

MOORMAN, C.; MINER, A. S. The impact of organizational memory on new product performance and creativity. Journal of Marketing Research, v.XXXIV, February, 1997, p. 91-106. 
NAKANO, D. N. Gestão do Conhecimento em Redes Interempresariais. In: AMATO Neto, J. Redes entre Organizações. São Paulo: Atlas, 2005.

NAKANO, D. N.; FLEURY,A. C. C. Organizational Learning, Learning organization and Knowledge Management models: a comparison. In: RIBEIRO et al. Production and Distribution Challenges for the 21st Century. VI International Conference on Industrial Engineering and Operations Management, Proceedings. Salvador: ABEPRO, 2001, p. 199-208.

NONAKA, I. A dynamic theory of organizational knowledge creation. Organization Science, v.5, n.1, February 1994, p.14-37.

NONAKA, I.; KONNO, N. The concept of "Ba": building a foundation for knowledge creation. California Management Review, v.40, n.3, spring 1998, p. 40-54.

NONAKA, I.; TAKEUCHI, H.The Knowledge-creating Company. New York: Oxford University Press, 1995, 284p.

O’DELL, C.; GRAYSON, C. J. If only we knew what we know: identification and transfer of internal best practices. California Management Review, v.40, n.3, spring 1998, p. 154-174.

OLIVERA, F. Memory systems in organizations: an empirical investigation of mechanisms for knowledge collection, storage and access. Journal of Management Studies, v.37, n.6, sept 2000, p. 811-832.

OLIVEIRA Jr., M. M. Competências Essenciais e Conhecimento na Empresa. In: FLEURY, M. T. L. e OLIVEIRA Jr., M. M. (org.) Gestão Estratégica do Conhecimento. São Paulo: Atlas, 2001.

PABLOS, P. O. Knowledge management and organizational learning: Typologies of knowledge strategies in the Spanish Manufacturing Industry from 1995 to 1999. Journal of Knowledge Management, v.6, n.1, 2002.

PARK, Y.; KIM, S. Linkage between knowledge management and R\&D management. Journal of Knowledge Management, v. 9, n. 4, 2005.

POLANYI, M. Tacit knowledge. In: PRUZAK, L. (ed.) Knowledge in Organizations. Newton, MA: Butterworth-Heinemann, 1997.

RONCHI, S.; CHAPMAN, R.; CORSO, M. Knowledge management in continuous product innovation: a contingent approach. International Journal of Technology Management, v. 26, n. 8, 2003. management in practice. California Management Review, v.40, n.3, spring 1998, p.80-9.

SCHNEIDER, S. C.; ANGELMAR, R. Cognition in organizational analysis: who's minding the store? Organization Studies, v.14, n.3, 1993.

SCHULTZE, U. e STABELL, C. Knowing What You Don't Know? Discourses and Contradictions in Knowledge Management Research. Journal of Management Studies, n. 4, v. 4, June 2004.

SENGE, P. M. The Fifth Discipline. New York: Doubleday/Currency, 1990, 424p.

SHRIVASTAVA, P. A typology of organizational learning systems. Journal of Management Studies, v.20, n.1, 1983, p.7-28.

STEWART, T. A. Intellectual Capital. New York: Doubleday / Currency, 1997. 278 p.

TAN, J. Managing knowledge - how to do it - a practical case study. The British Journal of Administrative Management, n.12, Mar/Apr 2000.

TEECE, D. J. Research directions for knowledge management. California Management Review, v.40, n.3, 1998, p. 289-292.

THOMPSON, M. P. A.; WALSHAM, G. Placing Knowledge Management in Context. Journal of Management Studies, v.41, n.5, July 2004.

VON KROGH, G. Care in knowledge creation. California Management Review, v.40, n.30, 1998, p. 133-53.

VON KROGH, G.; ROOS, J. and SLOCUM, K. An essay on corporate epistemology. Strategic Management Journal, v.15, 1994, p.53-17.

WALSH, J. P. Managerial and organizational cognition: notes from a trip down memory lane. Organization Science, v.6, n.3, May-June 1995, p. 280-321.

WALSH, J. P.; UNGSON, G. R. Organizational memory. The Academy of Management Review, v.16, n.1, 1991, p.57-91.

WATKINS, K. E.; MARSICK, V. J. Sculpting the Learning Organization. San Francisco: Jossey Bass, 1993, 298p. 
